\title{
Effect of bone marrow mesenchymal stem cells from blastic phase chronic myelogenous leukemia on the growth and apoptosis of leukemia cells
}

\author{
YUXIANG HAN ${ }^{1,2 *}$, YING WANG ${ }^{1 *}$, ZHENG XU ${ }^{1}$, JIE LI $^{1}$, JINGCI YANG $^{1}$, \\ YAN LI ${ }^{1}$, YINTAO SHANG ${ }^{1}$ and JIANMIN LUO ${ }^{1}$ \\ Departments of ${ }^{1}$ Hematology, and ${ }^{2}$ Immunology and Rheumatology, The Second Hospital, \\ Hebei Medical University, Shijiazhuang, Hebei 050000, P.R. China \\ Received February 24, 2013; Accepted May 8, 2013
}

DOI: $10.3892 /$ or.2013.2518

\begin{abstract}
Chronic myelogenous leukemia (CML) has a typical progressive course with transition from a chronic phase to a terminal blast crisis phase. However, the mechanisms that lead to disease progression remain unclear. Bone marrow mesenchymal stem cells (BMMSCs) play important roles in maintaining the bone marrow microenvironment. In the present study, the biological characteristics of BMMSCs were determined including proliferation, apoptosis and secretion of cytokines during blastic phase CML (CML-Bp). The effect of BMMSCs in CML-Bp on K562 human CML cells and the CML-Bp original generation leukemia cells were also explored. Our results showed that CML-Bp BMMSCs protect tumor cells and increase their anti-apoptotic ability through regulating the expression of apoptosis-related proteins and activating the Wnt pathway.
\end{abstract}

\section{Introduction}

Chronic myelogenous leukemia (CML) is a malignancy arising from hematopoietic stem cells. CML has a typical progressive course with transition from a chronic phase to a terminal blast crisis phase $(1,2)$. The therapeutic effects following treatment of blastic phase CML (CML-Bp) are currently disappointing. Prolonging the chronic phase and preventing the blastic phase constitute the main goals of CML treatment. However, the mechanisms that lead to disease progression remain unclear.

Bone marrow mesenchymal stem cells (BMMSCs) constitute a subset of non-hematopoietic adult stem cells originating

Correspondence to: Dr Jianmin Luo, Department of Hematology, The Second Hospital, Hebei Medical University, Shijiazhuang, Hebei 050000, P.R. China

E-mail: luojianmin2012@yahoo.com.cn

${ }^{*}$ Contributed equally

Key words: chronic myelogenous leukemia, blastic phase, mesenchymal stem cell, chronic phase from the mesoderm $(3,4)$. BMMSCs possess a self-renewal ability and are characterized by multilineage differentiation into not only mesoderm-lineage, such as chondrocytes, osteocytes, adipocytes, myocytes and astrocytes, but also ectodermic and endodermic cells (5-10). The bone marrow microenvironment supports and regulates the proliferation and differentiation of hematopoietic cells. BMMSCs are rare residents of the bone marrow microenvironment, but play important roles in maintaining the bone marrow microenvironment. Abnormal hematopoiesis in CML is related, at least in part, to abnormalities in the hematopoietic cells themselves leading to abnormal interactions between CML progenitors and the marrow microenvironment. The role of the microenvironment itself in CML has not been well characterized. It was found that BMMSCs regulate the proliferation of leukemia cells through the secretion of cytokines (11). In addition, the interaction between leukemia cells and BMMSCs affects the hematopoietic microenvironment (12).

In the present study, the biological characteristics of BMMSCs were determined including proliferation, apoptosis and the secretion of cytokines during CML-Bp. The effects of BMMSCs in CML-Bp on human CML K562 cells and the CML-Bp original generation leukemia cells were also investigated.

\section{Materials and methods}

Patients and healthy donors. Thirty CML patients who were treated at the Department of Hematology, The Second Hospital, Hebei Medical University (Shijiazhuang, China) were included in the present study. These patients included 20 (12 males, 9 females) chronic phase CML (CML-Cp) patients and 10 (6 males, 4 females) CML-Bp patients. Ten healthy donors were included in the study as the control group. All the individuals provided written informed consent prior to enrollment. This study was approved by the Medical Ethics Committee of the Second Hospital of Hebei Medical University.

Cell culture. For primary CML-Bp leukemia cells, after informed consent was obtained from all the subjects, blood mononuclear cells were collected by bone marrow aspiration 
Table I. List of primers used in RT-PCR.

\begin{tabular}{|c|c|c|}
\hline Gene & Primer & Size (bp) \\
\hline IL-6 & $\begin{array}{ll}\text { F: } & \text { 5'-CACACAGACAGCCACTCACC-3' } \\
\text { R: } & \text { 5'-TCTTTGGAAGGTTCAGGTTGT-3' }\end{array}$ & 330 \\
\hline IL-12 & $\begin{array}{ll}\text { F: } & \text { 5'-TTCTCCCTGACATTCTGCG-3' } \\
\text { R: } & \text { 5'-CCATTCGCTCCAAGATGAG-3' }\end{array}$ & 356 \\
\hline SCF & $\begin{array}{ll}\text { F: } & \text { 5'-GGAAAGAAGACAACGACACG-3' } \\
\text { R: } & \text { 5'-GGGTCAGGAATAAACCTCAAGT-3' }\end{array}$ & 143 \\
\hline TPO & $\begin{array}{ll}\text { F: } & \text { 5'-GACCTCCGAGTCCTCAGTAAAC-3' } \\
\text { R: } & \text { 5'-GAATGTCCTGTGCCTTGGT-3' }\end{array}$ & 125 \\
\hline G-CSF & $\begin{array}{ll}\mathrm{F}: & \text { 5'-GACCCAAGAGCAGTTTCC-3' } \\
\mathrm{R}: & \text { 5'-AGTCACAGCGGAGATAGTGC-3' }\end{array}$ & 138 \\
\hline BCL/ABL & $\begin{array}{ll}\text { F: } & \text { 5'-GCTTCTCCCTGACATCCGTG-3' } \\
\text { R: } & \text { 5'-CGAGCGGCTTCACTCAGACC-3' } \\
\text { F: } & \text { 5'-CTCCAGACTGTCCACAGCATTCCG-3' } \\
\text { R: } & \text { 5'-CAGACCCTGAGGCTCAAAGTCAGA-3' }\end{array}$ & 165 \\
\hline GAPDH & $\begin{array}{ll}\text { F: } & \text { 5'-TGAACGGGAAGCTCACTGG-3' } \\
\text { R: } & \text { 5'-GCTTCACCACCTTCTTGATGTC-3' }\end{array}$ & 120 \\
\hline
\end{tabular}

RT-PCR, reverse transcription-polymerase chain reaction; IL, interleukin; SCF, stem cell factor; TPO, thombopoietin; G-CSF, granulocyte-colony stimulating factor.

(heparinized bone marrow). The heparinized bone marrow was diluted twice with phosphate-buffered saline (PBS). Nucleated cells were then isolated by density-gradient centrifugation and cultured in RPMI-1640 supplemented with $100 \mathrm{U} / \mathrm{ml}$ penicillin, $100 \mathrm{U} / \mathrm{ml}$ streptomycin and $10 \%$ fetal bovine serum (FBS) at $37^{\circ} \mathrm{C}$ with $5 \% \mathrm{CO}_{2}$.

K562 cells, obtained from a patient with chronic myeloid leukemia in blast crisis, were constantly preserved in our laboratory. The culture conditions were identical to primary CML-Bp leukemia cells.

The blood mononuclear cells were isolated by density-gradient centrifugation, incubated and cultured in BMMSC culture medium supplemented with $100 \mathrm{U} / \mathrm{ml}$ penicillin, $100 \mathrm{U} / \mathrm{ml}$ streptomycin and $10 \% \mathrm{FBS}$ at $37^{\circ} \mathrm{C}$ with $5 \% \mathrm{CO}_{2}$. The medium was replaced twice per week and nonadherent cells were discarded. When BMMSCs were 80-90\% confluent, the cells were digested with trypsin and harvested in the medium. BMMSCs of the 4 th or 5 th passage were used in this study.

MTT assay. The cells were seeded at a density of $5 \times 10^{3} /$ well in 96-well plates and cultured in a $5 \% \mathrm{CO}_{2}$ incubator. The cells were treated uner the indicated conditions, such as with adriamycin (ADM; Zhejiang Pharmaceutical Co., Ltd., China). For the indicated time periods, $20 \mu \mathrm{l}$ of MTT solution (Amresco, Solon, OH, USA) was added to each well. After $4 \mathrm{~h}$ of incubation, the medium was discarded and $150 \mu$ l of dimethyl sulfoxide (DMSO) was added into each well. The cells were shaken in the dark for $10 \mathrm{~min}$. The absorbance reading for each well was performed at $490 \mathrm{~nm}$ using a microplate reader. Each assay was repeated at least 3 times.
Apoptosis assay by flow cytometry. The cells were treated with ADM or Dickkopf-1 (DKK1, recombinant human DKK1 protein; R\&D Systems, Minneapolis, MN, USA). Both suspension and adherent cells were collected, and washed twice with ice-cold PBS. The cells were then suspended in $200 \mu \mathrm{l}$ of binding buffer and $10 \mu \mathrm{l}$ of Annexin V-FITC for $15 \mathrm{~min}$ in the dark. Subsequently, $300 \mu \mathrm{l}$ of binding buffer and $5 \mu \mathrm{l}$ of propidium iodide (PI) were added to each sample. Finally, the cells were analyzed using BD FACSDiva flow cytometry (BD FACS Canto $^{\mathrm{TM}}$ II) with CellQuest software.

RNA extraction and reverse transcription-polymerase chain reaction $(R T-P C R)$ analysis. RT-PCR was used to determine the expression of BCR/ABL, thombopoietin (TPO), interleukin (IL)-6, IL-12, stem cell factor (SCF) and granulocyte-colony stimulating factor (G-CSF). Total RNA was extracted using TRIzol reagent (Invitrogen, Carlsbad, CA, USA) according to the manufacturer's instructions. The primers used are listed in Table I. The conditions for PCR were as follows: denaturation at $94^{\circ} \mathrm{C}$ for $4 \mathrm{~min}$, denaturation at $94^{\circ} \mathrm{C}$ for $50 \mathrm{sec}$, annealing at $52^{\circ} \mathrm{C}$ for $45 \mathrm{sec}$, and extension at $72^{\circ} \mathrm{C}$ for $45 \mathrm{sec}, 35$ cycles, and a final $10 \mathrm{~min}$ at $72^{\circ} \mathrm{C}$. PCR products were visualized by gel electrophoresis on $1.5 \%$ agarose $(\mathrm{w} / \mathrm{v})$ gel, and then viewed using an ultraviolet photometry (UVP) bioimaging system.

Western blot analysis. Total protein was extracted and quantified according to the manufacturer's protocol. Each equal amount of protein was loaded on sodium dodecyl sulfate-polyacrylamide gel at $100 \mathrm{~V}$ for $2 \mathrm{~h}$, and then the protein was transferred to polyvinylidene fluoride (PVDF)-membranes. The membranes were blocked in $5 \%$ fat-free milk at room 

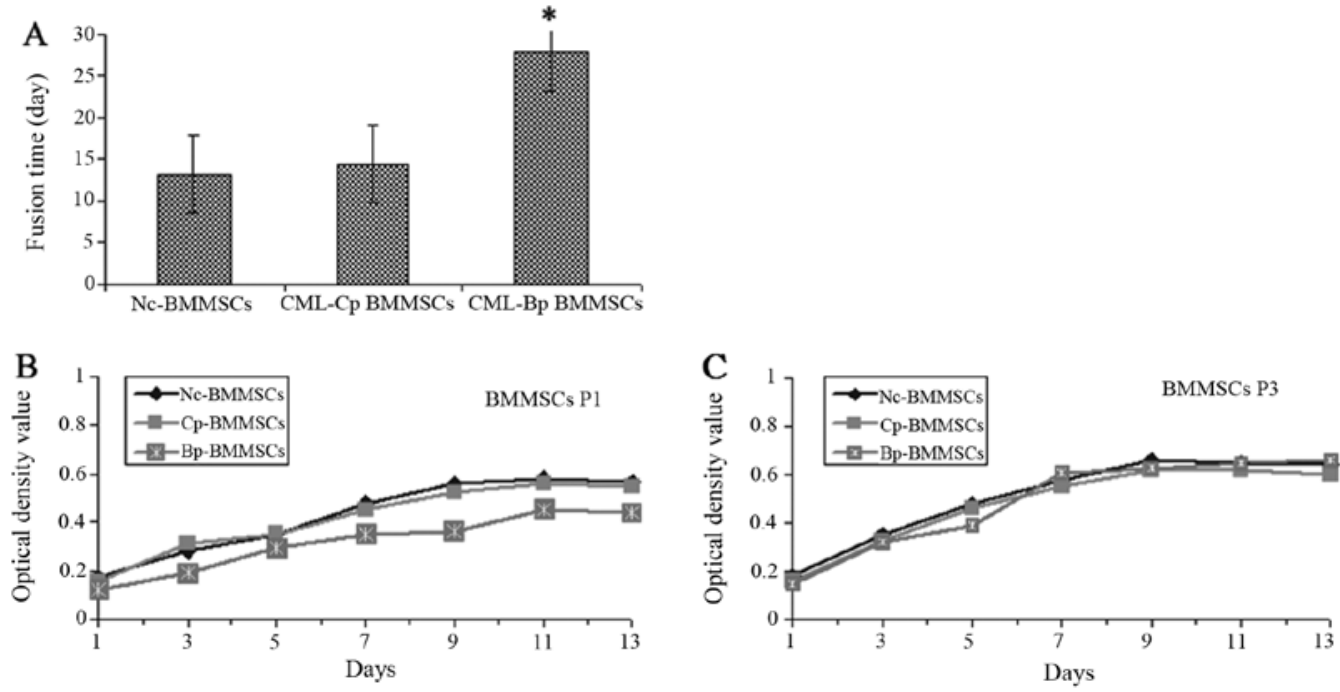

Figure 1. Growth, proliferation and passage of CML-Bp BMMSCs. (A) Fusion time (90\%) of primary CML-Bp BMMSCs. *P<0.05 compared with Nc-BMMSCs and CML-Cp BMMSCs. (B and C) Growth curves of the different BMMSCs (P1 and P3). CML, chronic myelogenous leukemia; Cp, chronic phase; Bp, blastic phase; BMMSCs, bone marrow mesenchymal stem cells.

temperature for $2 \mathrm{~h}$, and the blots were stained with specific primary antibodies, including anti-bcl-2 (BioWorld, Atlanta, GA, USA), anti-bax (BioWorld), anti-active caspase-3 (Cell Signaling Technology, Inc., Danvers, MA, USA), anti- $\beta$-catenin (Santa Cruz Biotechnology, Inc., Santa Cruz, CA, USA) and anti-GAPDH antibodies (Santa Cruz Biotechnology, Inc.) overnight at $4^{\circ} \mathrm{C}$. The membranes were then washed and incubated with goat anti-rabbit HRP-IgG (Santa Cruz Biotechnology, Inc.) for $1 \mathrm{~h}$ at room temperature, and observed with a chemiluminescent substrate. Bound immunoglobulins were removed from the membranes by washing twice with Restore $^{\mathrm{TM}}$ Western Blot Stripping Buffer, and the signal was visualized by enhanced chemiluminescence and detected using the ChemiDoc XRS+ system (Bio-Rad, Hercules, CA, USA); the signal was analyzed by the Image Lab (ECL). GAPDH was used as an internal control.

Statistical analysis. Data are presented as means \pm standard deviation (SD). All the statistical analyses were performed using SPSS 13.0 software. The statistical analysis of results was carried out using Student's t-test and one-way ANOVA analysis. $\mathrm{P}<0.05$ was considered to indicate a statistically significant difference.

\section{Results}

Growth, proliferation and passage of CML-Bp BMMSCs. As shown in Fig. 1A, the $90 \%$ fusion time of primary CML-Bp BMMSCs was significantly longer compared with Nc-BMMSCs and CML-Cp BMMSCs (27.75 \pm 2.29 vs. $13.18 \pm 1.31$ and $14.46 \pm 1.56, \mathrm{P}<0.05)$. CML-Bp BMMSCs at first passage grew slowly with a few colonies and adherent cells (Fig. 1B). At the third generations, the cell proliferative capacity of CML-Bp BMMSCs was enhanced, similarly to CML-Cp BMMSCs and Nc-BMMSCs (Fig. 1C).

Apoptotic rate of CML-Bp BMMSCs. As shown in Fig. 2, the apoptotic rate of Nc-BMMSCs, CML-Cp BMMSCs and
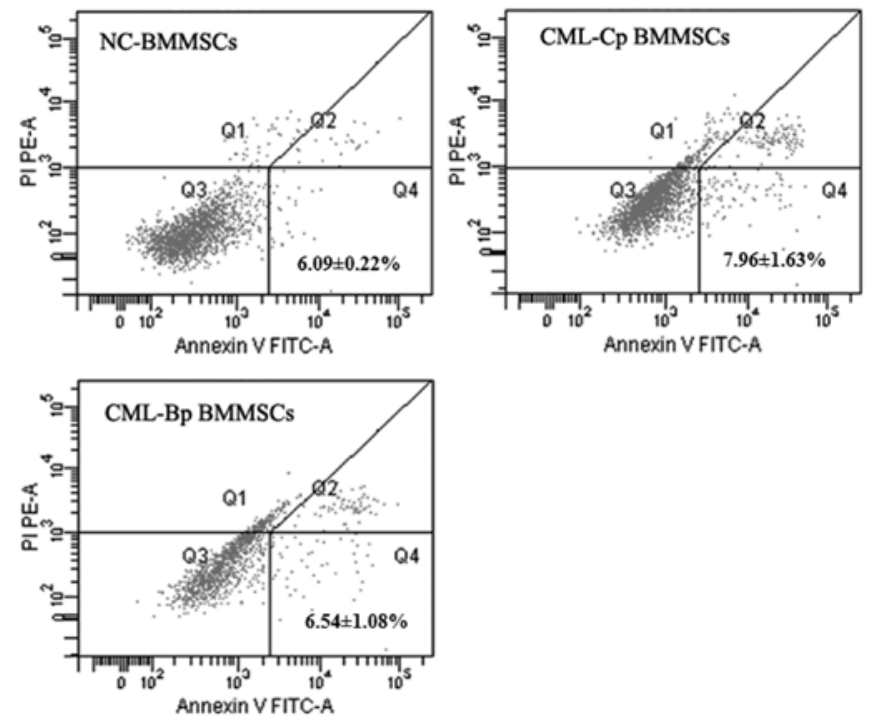

Figure 2. Apoptotic rate of BMMSCs detected using flow cytometry. ${ }^{*} \mathrm{P}>0.05$ compared with Nc-BMMSCs and Cp-BMMSCs. CML, chronic myelogenous leukemia; $\mathrm{Cp}$, chronic phase; $\mathrm{Bp}$, blastic phase; BMMSCs, bone marrow mesenchymal stem cells.

CML-Bp BMMSCs was 6.09 $\pm 0.22,7.96 \pm 1.63$ and $6.54 \pm 1.08 \%$, respectively. No significant difference was observed among the three groups $(\mathrm{P}>0.05)$.

Expression of Bcr/abl fusion gene and cytokine secretion. As shown in Fig. 3A, the Bcr/abl fusion gene was expressed in human CML K562 cells and CML-Bp primary leukemia cells, while it was not expressed in CML-Bp BMMSCs, Nc-BMMSCs and CML-Cp BMMSCs. The expression of TPO, IL-6, IL-12 and SCF in the CML-Bp BMMSC group was significantly lower compared with the CML-Cp BMMSC group $(\mathrm{P}<0.05)$. However, G-CSF secretion was not significantly different between these two groups $(\mathrm{P}>0.05)$ (Fig. 3B and C). 
A

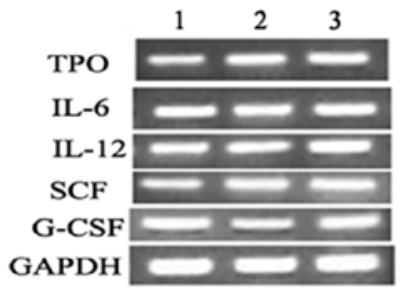

$\mathrm{C}$

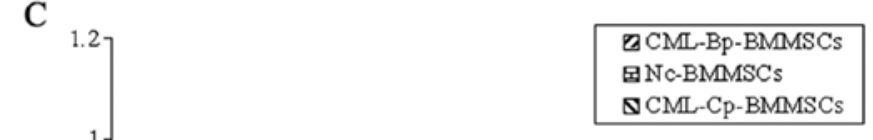

B

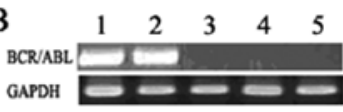

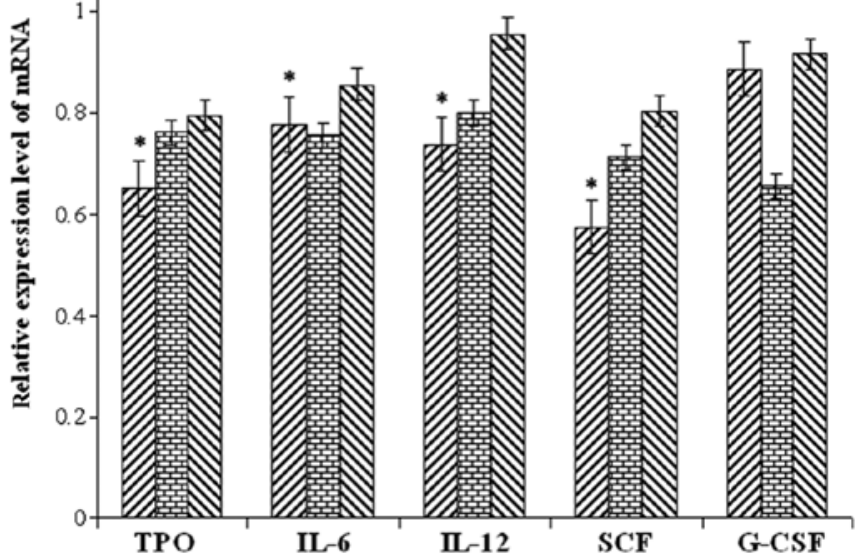

Figure 3. Expression of BCR/ABL and cytokines detected using RT-PCR. (A and C) Expression of TPO, IL-6, IL-12, SCF and G-CSF mRNA detected by RT-PCR. Lane 1, CML-Bp-BMMSCs; lane 2, Nc-BMMSCs; lane 3, CML-Cp-BMMSCs. "P $<0.05$ compared with CML-Cp BMMSCs. (B) Expression of BCR/ABL mRNA detected by RT-PCR. Lane 1, K562 cells; lane 2, CML-Bp primary leukemia cells; lane 3, Nc-BMMSCs; lane 4, CML-Cp BMMSCs; lane 5, CML-Bp BMMSCs. CML, chronic myelogenous leukemia; $\mathrm{Cp}$, chronic phase; $\mathrm{Bp}$, blastic phase; BMMSCs, bone marrow mesenchymal stem cells.

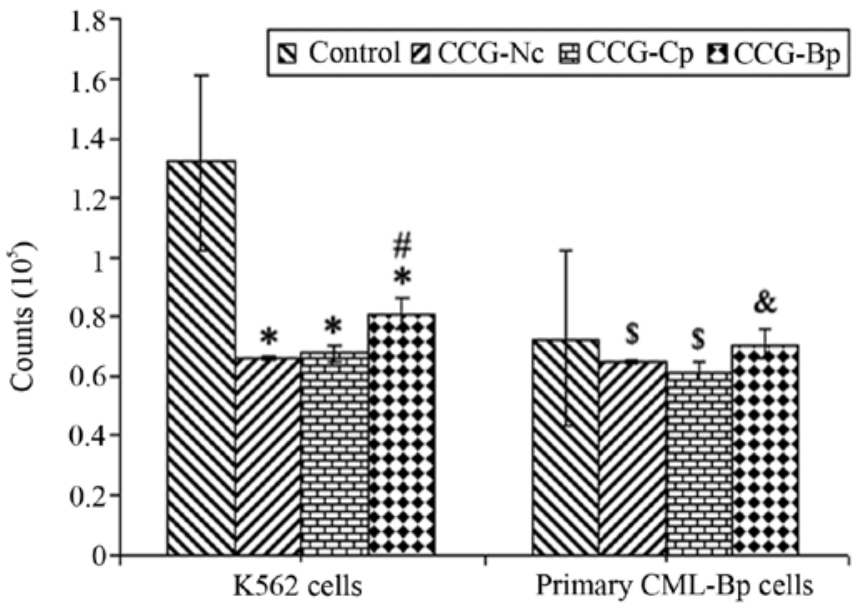

Figure 4. Proliferation of K562 cells and primary CML-Bp cells $48 \mathrm{~h}$ following co-culture with or without BMMSCs. ${ }^{*} \mathrm{P}<0.05$ compared with K562 cells; ${ }^{*} \mathrm{P}<0.05$ compared with CCG-Nc and CCG-Cp cells; ${ }^{\$} \mathrm{P}<0.05$ compared with primary CML-Bp leukemia cells; ${ }^{\&} \mathrm{P}>0.05$ compared with primary CML-Bp leukemia cells. CML, chronic myelogenous leukemia; $\mathrm{Cp}$, chronic phase; Bp, blastic phase; BMMSCs, bone marrow mesenchymal stem cells.

Effects of BMMSCs on the proliferation of K562 and primary $C M L-B p$ cells. As shown in Fig. 4, K562 cells and primary CML-Bp cells were co-cultured with Nc-BMMSCs (CCG-Nc

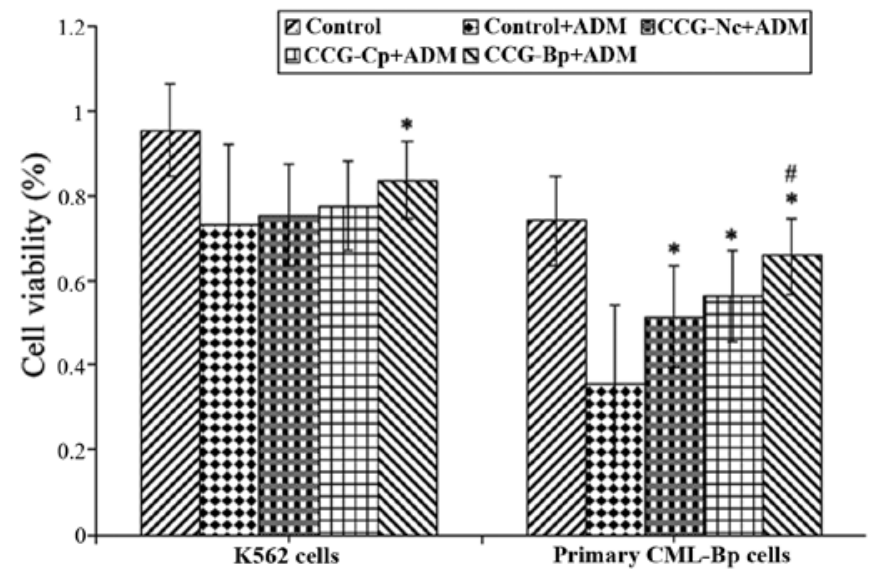

Figure 5. Cell viability of K562 cells and primary CML-Bp leukemia cells $48 \mathrm{~h}$ following co-culture with or without BMMSCs and treatment with adriamycin (ADM). ${ }^{*} \mathrm{P}<0.05$ compared with control cells; ${ }^{\#} \mathrm{P}<0.05$ compared with CCG-Nc and CCG-Cp cells. CML, chronic myelogenous leukemia; $\mathrm{Cp}$, chronic phase; Bp, blastic phase; BMMSCs, bone marrow mesenchymal stem cells.

group), CML-Bp BMMSCs (CCG-Bp group) and CML-Cp BMMSCs (CCG-Cp group) for $48 \mathrm{~h}$, respectively. The ratio of BMMSCs to K562 or primary CML-Bp cells was 1:10. The growth of K562 cells was significantly decreased following co-culture with BMMSCs, with a slight decrease in the CML-Bp BMMSC group $(\mathrm{P}<0.05)$. However, the growth of primary CML-Bp cells was not significantly altered following co-culture with BMMSCs, with a slight decrease in the CML-Bp BMMSC group ( $\mathrm{P}>0.05)$. These results to some extent indicate that CML-Bp BMMSCs only slightly inhibited K562 cell proliferation, while they did not inhibit the growth of primary CML-Bp leukemia cells.

Effects of BMMSCs on the proliferation of K562 and primary $C M L-B p$ cells following ADM treatment. As shown in Fig. 5, K562 and primary CML-Bp cells were co-cultured with Nc-BMMSCs, CML-Bp BMMSCs and CML-Cp BMMSCs for $48 \mathrm{~h}$, respectively. The cells were then treated with ADM for $48 \mathrm{~h}$. The cell viability of the CML-Bp BMMSC group was significantly increased when compared with the ADM control group $(83.78 \pm 5.17$ vs. $73.13 \pm 2.42 \%, \mathrm{P}<0.05)$. The cell viability of the Nc-BMMSC and CML-Cp BMMSC groups was $75.45 \pm 3.27$ and $77.56 \pm 3.11 \%$, respectively, which was not significantly different compared with the ADM control group $(\mathrm{P}>0.05)$. Thus, these data indicate that CML-Bp BMMSCs, but not CML-Cp BMMSCs or Nc BMMSC, protected K562 cells against apoptosis. In addition, CML-Bp BMMSCs were shown to protect the primary CML-Bp leukemia cells.

Effects of BMMSCs on ADM-induced apoptosis of K562 and primary $C M L-B p$ cells. As shown in Fig. 6, K562 cells were co-cultured with Nc-BMMSCs, CML-Bp BMMSCs and CML-Cp BMMSCs for $48 \mathrm{~h}$, respectively. The cells were then treated with ADM for $48 \mathrm{~h}$. The apoptotic rate of the Nc-BMMSC, CML-Cp BMMSC and CML-Bp BMMSC groups was $19.9 \pm 0.82,18.5 \pm 1.63$ and $13.4 \pm 2.15 \%$, respectively, which was significantly decreased compared with the apoptotic rate of the control group $(23.1 \pm 2.45 \%, \mathrm{P}<0.05)$. The 

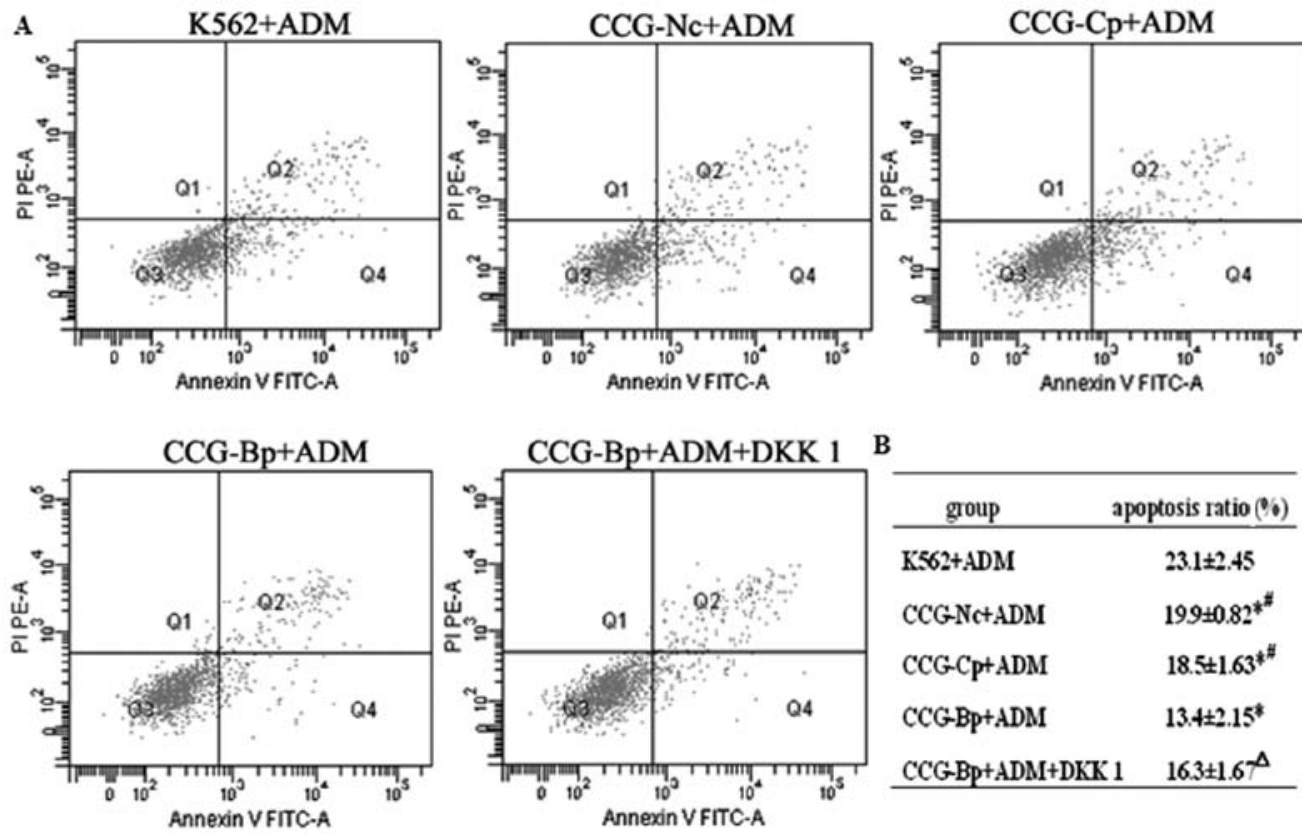

Figure 6. Apoptotic rate of K562 cells $48 \mathrm{~h}$ following co-culture with or without bone marrow mesenchymal stem cells (BMMSCs) and treatment with adriamycin (ADM). ${ }^{*} \mathrm{P}<0.05$ compared with $\mathrm{K} 562+\mathrm{ADM}$ group; ${ }^{*} \mathrm{P}<0.05$ compared with $\mathrm{CCG}-\mathrm{Bp}+\mathrm{ADM}$ group; ${ }^{\triangle} \mathrm{P}<0.05$ compared with $\mathrm{CCG}-\mathrm{Bp}+\mathrm{ADM}$ group.

A
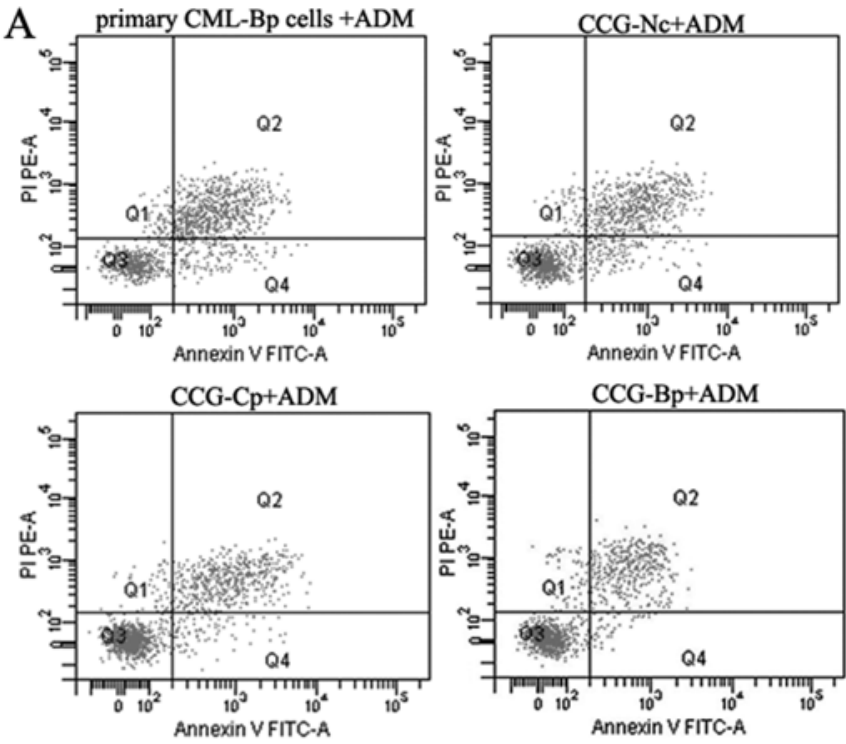

B

\begin{tabular}{cc}
\hline group & apoptosis ratio(\%) \\
\hline primary CML-Bp cells+ADM & $60.9 \pm 2.52$ \\
CCG-Nc+ADM & $45.9 \pm 2.82^{*}$ \\
CCG-Cp+ADM & $40.1 \pm 5.63^{*}$ \\
CCG-Bp+ADM & $30.5 \pm 2.33^{*}$
\end{tabular}

Figure 7. Apoptotic rate of primary CML-Bp leukemia cells $48 \mathrm{~h}$ following co-culture with or without bone marrow mesenchymal stem cells (BMMSCs) and treatment with adriamycin (ADM). ${ }^{*} \mathrm{P}<0.05$ compared with primary cells+ADM group; ${ }^{*} \mathrm{P}<0.05$ compared with $\mathrm{CCG}-\mathrm{Bp}+\mathrm{ADM}$ group.

most significantly decreased apoptotic rate was observed in the CML-Bp BMMSC group.

As shown in Fig. 7, primary CML-Bp leukemia cells were co-cultured with Nc-BMMSCs, CML-Bp BMMSCs

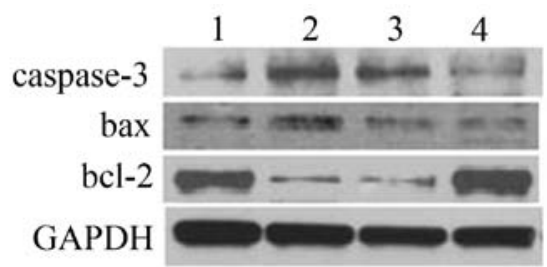

Figure 8. Expression of bcl-2, bax and caspase-3 proteins in K562 cells. Lane 1, K562 alone; lane 2, K562+ADM; lane 3, CCG-Cp+ADM; lane 4, CCG-Bp+ADM.

and CML-Cp BMMSCs for $48 \mathrm{~h}$, respectively. The cells were then treated with ADM for $48 \mathrm{~h}$. The apoptotic rate of the Nc-BMMSC, CML-Cp BMMSC and CML-Bp BMMSC groups was $45.9 \pm 2.82,40.1 \pm 5.63$ and $30.5 \pm 2.33 \%$, respectively, which was significantly decreased compared with the control group $(60.9 \pm 2.52 \%, \mathrm{P}<0.05)$. The most significantly decreased apoptotic rate was observed in the CML-Bp BMMSC group. These results indicate that CML-Bp BMMSCs reduced ADM-induced leukemia cell apoptosis.

Expression of bcl-2, bax and caspase-3. The expression of caspase- 3 and bax of K562 cells at the protein level was significantly increased following ADM treatment, while the expression of bcl-2 was significantly decreased (Fig. 8), suggesting that K562 cells underwent apoptosis in response to $\mathrm{ADM}$ treatment. In the CML-Bp BMMSC group, the expression of caspase- 3 and bax were significantly decreased, while bcl-2 expression was significantly increased compared with the ADM control group. There was no significant change in the CML-Cp BMMSC group compared with the ADM control group. These results suggest that CML-Bp BMMSCs inhibited ADM-induced K562 cell apoptosis. 


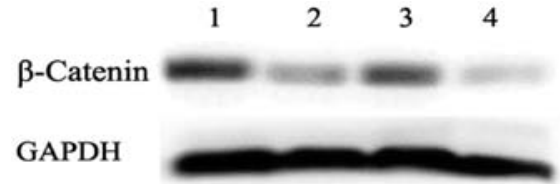

Figure 9. Expression of $\beta$-catenin protein in K562 cells detected by western blot analysis. Lane 1, K562 alone; lane 2, K562+ADM; lane 3, CCG-Bp+ADM; lane 4, CCG-Bp+ADM+DKK1. ADM, adriamycin; Bp, blastic phase; DKK1, Dickkopf-1.

$D K K 1$ protein reverses the protective effect of $C M L-B p$ BMMSCs on K562 cells. DKK1 is a secretory antagonist of the $\mathrm{Wnt} / \beta$-catenin signaling pathway and has been shown to play a crucial role in carcinogenesis. Our results showed that the ADM-induced cell apoptosis of K562 cells in the CML-Bp BMMSC group recovered after DKK1 treatment $(13.4 \pm 2.15$ vs. $16.3 \pm 1.67 \%, \mathrm{P}<0.05$; Fig. 6$)$. The results from western blot analysis showed that $\beta$-catenin expression of K562 cells was significantly decreased following ADM treatment. In the ADM-treated CML-Bp BMMSC group, the expression of $\beta$-catenin was downregulated following DKK1 treatment (Fig. 9). These results to some extent suggest that CML-Bp BMMSCs antagonize ADM-induced K562 cell apoptosis, potentially through the activation of the Wnt pathway.

\section{Discussion}

To elucidate the correlation between BMMSCs and CML-Bp, the determination of the biological characteristics of BMMSCs from CML-Bp and their effect on leukemia cells is required. In the present study, we found that the growth of primary CML-Bp BMMSCs was significantly slower when compared with the growth of CML-Cp BMMSCs. After three passages, the proliferation of CML-Bp BMMSCs was increased. The spontaneous apoptosis of CML-Bp BMMSCs was similar to that of the Nc-BMMSCs and CML-Cp BMMSCs. Our results showed that the expression levels of IL-6, IL-12, SCF and TPO in CML-Bp BMMSCs were significantly lower when compared with CML-Cp BMMSCs $(\mathrm{P}<0.05)$. SCF has a positive regulatory effect on hematopoiesis (13), and IL-6 affects the early proliferation and differentiation of bone marrow hematopoietic stem cells (14). The downregulation of IL-12 has been suggested to result in defects of the immune surveillance system of tumor cells in leukemia patients, and the IL-12 expression levels to increase after treatment (15). These results suggested that the expression levels of certain cytokines were altered during the proliferation of CML-Bp BMMSCs. In addition, BCR/ABL expression was not observed in CML-Bp BMMSCs and CML-Cp BMMSCs. BCR/ABL expression is a marker of CML malignant clones, suggesting that $\mathrm{CML}$ malignant clones might not accumulate during CML-Bp.

It has been shown that BMMSCs regulate the proliferation of leukemia cells, displaying an inhibitory or promotive effect on the growth of tumor cells (16-18). Contradictory results might have been obtained due to the heterogeneity of MSCs and the different response to various tumor cells under different experimental conditions. The present study demonstrated that CML-Cp BMMSCs significantly inhibited the growth of leukemia K562 cells in the absence of chemotherapeutic agents, while CML-Bp BMMSCs had a weak inhibitory effect on K562 cells and no inhibitory effect on primary CML-Bp leukemia cells. However, no promotive effect of CML-Bp BMMSCs on the proliferation of tumor cells was observed.

BMMSCs constitute an important part of the bone marrow microenvironment and play an important role in the development and drug-resistance of hematopoietic malignancies. It has been reported that imatinib resistance is associated with the protective effect of the bone marrow microenvironment on leukemia stem cells (19). Nefedova et al (20) and Konopleva et al (21) found that BMMSCs are involved in the chemotherapy resistence of leukemia cells, a fact that led to the investigation of the protective effect of BMMSCs on tumor cells. The results of the present study showed that BMMSCs decreased the ADM-induced K562 apoptosis, suggesting that BMMSCs protect K562 cells and antagonize ADM-induced apoptosis. CML-Bp BMMSCs were found to have the strongest protective effect on leukemia cells when compared with CML-Cp BMMSCs and Nc-BMMSCs.

Blockade of tumor cell apoptosis is an important process underlying chemotherapy resistance. Bcl-2, Bax, survivin, p53 and $\mathrm{C}$-myc are involved in the regulation of the anti-apoptotic ability of leukemia cells, in addition to the P38MAPK, Wnt, PI3K/Akt and NF- $\mathrm{B}$ pathways (22-24). Our results showed that CML-Bp BMMSCs upregulated the expression of bcl-2, downregulated the expression of bax and active caspase- 3 protein, suggesting that CML-Bp BMMSCs protected K562 cells against apoptosis by inhibiting the apoptotic pathway. The Bcl-2 family was found to be involved in this process.

However, in the present study, DKK1 increased the apoptosis of K562 cells, and downregulated the expression of $\beta$-catenin, suggesting that CML-Bp BMMSCs activate the Wnt pathway and reduce the apoptosis of K562 cells. As a negative regulator of the Wnt pathway, DKK1 inhibited the activation of the Wnt pathway in K562 cells, leading to increased apoptosis, further suggesting that the Wnt pathway is involved in this process. The essence of CML blastic change is the proliferation of original naive cells. CML blastic change has been suggested to be related to the activation of the Wnt pathway $(25,26)$. Wnt/ $\beta$-catenin, as an important self-renewal pathway (27), regulated the transcription of survivin, p53 and c-myc. Subsequent experiments showed that blocking the protective effect of BMMSCs, thereby restoring the sensitivity to chemotherapy drugs, could be used as a novel potential treatment strategy. However, DKK1 did not completely reverse the protective effect of BMMSCs on K562 cells, suggesting the involvement of additional protective mechanisms.

In conclusion, the levels of the cytokines secreted by CML-Bp BMMSCs were altered when compared with CML-Cp BMMSCs. CML-Bp BMMSCs protect tumor cells and increase the anti-apoptotic ability through regulating the expression of apoptosis-related proteins and activating the Wnt pathway. Further studies are needed in order to elucidate whether there is a correlation between CML-Bp BMMSCs and CML.

\section{References}

1. Uehara E, Takeuchi S, Yang Y, et al: Aberrant methylation in promoter-associated $\mathrm{CpG}$ islands of multiple genes in chronic myelogenous leukemia blast crisis. Oncol Lett 3: 190-192, 2012. 
2. Cortes $\mathbf{J}$ and Kantarjian $\mathrm{H}$ : How I treat newly diagnosed chronic phase CML. Blood 120: 1390-1397, 2012.

3. Deans RJ and Moseley AB: Mesenchymal stem cells: biology and potential clinical uses. Exp Hematol 28: 875-884, 2000.

4. Jiang Y, Jahagirdar BN, Reinhardt RL, et al: Pluripotency of mesenchymal stem cells derived from adult marrow. Nature 418 : 41-49, 2002.

5. Barry FP and Murphy JM: Mesenchymal stem cells: clinical applications and biological characterization. Int $\mathrm{J}$ Biochem Cel Biol 36: 568-584, 2004

6. Bianchi G, Borgonovo G, Pistoia V and Raffaghello L: Immunosuppressive cells and tumour microenvironment: focus on mesenchymal stem cells and myeloid derived suppressor cells. Histol Histopathol 26: 941-951, 2011.

7. Prockop DJ: Marrow stromal cells as stem cells for nonhematopoietic tissues. Science 276: 71-74, 1997.

8. Granero-Molto F, Weis JA, Longobardi L and Spagnoli A: Role of mesenchymal stem cells in regenerative medicine: application to bone and cartilage repair. Expert Opin Biol Ther 8: 255-268, 2008.

9. Salem HK and Thiemermann C: Mesenchymal stromal cells: current understanding and clinical status. Stem Cells 28 $585-596,2010$.

10. Dezawa M, Ishikawa H, Itokazu Y, et al: Bone marrow stromal cells generate muscle cells and repair muscle degeneration. Science 309: 314-317, 2005.

11. Oostendorp RA and Dörmer P: VLA-4-mediated interactions between normal human hematopoietic progenitors and stromal cells. Leuk Lymphoma 24: 423-435, 1997.

12. Colmone A, Amorim M and Pontier AL: Leukemic cells create bone marrow niches that disrupt the behavior of normal hematopoietic progenitor cells. Science 322: 1861-1865, 2008.

13. Duarte RF and Frank DA: SCF and G-CSF lead to the synergistic induction of proliferation and gene expression through complementary signaling pathways. Blood 96: 3422-3430, 2000.

14. Frick JS, Zahir N, Mulier M, et al: Colitogenic and non-colitogenic commensal bacteria differentially trigger DC maturation and Th cell polarization: an important role for IL-6. Eur J Immunol 36: 1537-1547, 2006.

15. Bandini G, Zuffa E, Rosti G, et al: Long-term outcome of adults with acute myelogenous leukaemia: results of a prospective, randomized study of chemotherapy with a minimal follow-up of 7 years. Br J Haematol 77: 486-490, 1991.
16. Zhu Y, Sun Z, Han Q, et al: Human mesenchymal stem cells inhibit cancer cell proliferation by secreting DKK-1. Leukemia 23: 925-933, 2009.

17. Moshaver B, van der Pol MA, Westra AH, et al: Chemotherapeutic treatment of bone marrow stromal cells strongly affects their protective effect on acute myeloid leukemia cell survival. Leuk Lymphoma 49: 134-148, 2008.

18. Gaundar SS, Bradstock KF and Bendall LJ: p38MAPK inhibitor attenuate cytokine production by bone marrow stromal cells and reduce stroma-mediated proliferation of acute lymphoblastic leukemia cells. Cell Cycle 8: 2975-2983, 2009.

19. Konopleva M and Andreeff M: Targeting the leukemia microenvironment. Curr Drug Targets 8: 685-701, 2007.

20. Nefedova Y, Landowski TH and Dalton WS: Bone marrow stromal-derived soluble factors and direct cell contact contribute to de novo drug resistance of myeloma cells by distinct mechanisms. Leukemia 17: 1175-1182, 2003.

21. Konopleva M, Konoplev S, Hu W, et al: Stromal cells prevent apoptosis of AML cells by up-regulation of anti-apoptotic proteins. Leukemia 16: 1713-1724, 2002

22. Yeung J, Esposito MT, Gandillet A, et al: $\beta$-Catenin mediates the establishment and drug resistance of MLL leukemic stem cells. Cancer Cell 18: 606-618, 2010.

23. Sheth K, Friel J, Nolan B and Bankey P: Inhibition of p38 mitogen activated protein kinase increases lipopolysaccharide induced inhibition of apoptosis in neutrophils by activating extracellular signal-regulated kinase. Surgery 130: 242-248, 2001

24. Grandage VL, Gale RE, Linch DC and Khwaja A: PI3-kinase/Akt is constitutively active in primary acute myeloid leukaemia cells and regulates survival and chemoresistance via NF-kappaB, Mapkinase and p53 pathways. Leukemia 19: 586-594, 2005.

25. Valencia A, Román-Gómez J, Cervera J, et al: Wnt signaling pathway is epigenetically regulated by methylation of Wnt antagonists in acute myeloid leukemia. Leukemia 23: 1658-1666, 2009.

26. Radich JP, Dai H, Mao M, et al: Gene expression changes associated with progression and response in chronic myeloid leukemia. Proc Natl Acad Sci USA 103: 2794-2799, 2006.

27. $\mathrm{Hu} \mathrm{Y,} \mathrm{Chen} \mathrm{Y,} \mathrm{Douglas} \mathrm{L} \mathrm{and} \mathrm{Li} \mathrm{S:} \mathrm{Beta-Catenin} \mathrm{is} \mathrm{essential} \mathrm{for}$ survival of leukemic stem cells insensitive to kinase inhibition in mice with BCR-ABL-induced chronic myeloid leukemia. Leukemia 23: 109-116, 2009. 